\title{
PÁNCREAS HETEROTÓPICO COMO CAUSA DE INVAGINACIÓN INTESTINAL: PRIMER CASO REPORTADO EN EL PERÚ
}

\author{
Carlos Bazán Zender ${ }^{1, a}$, Luis Reyes Coloma ${ }^{1, b}$, José Luis León Cueto ${ }^{1, b}$, Giancarlo Revoredo Palacios ${ }^{1, a}$, \\ Javier Arias Stella Castillo, ${ }^{1, c}$, Alonso Pezo ${ }^{1, d}$
}

\begin{abstract}
RESUMEN
El páncreas heterotópico $(\mathrm{PH})$ es una condición rara en la población pediátrica. Los casos de $\mathrm{PH}$ que conllevan a una invaginación ileal, son infrecuentes en niños y muy raramente reportados, usualmente mostrándose con síntomas de obstrucción intestinal. Se presenta el caso de un paciente varón de un año de edad, con una historia crónica de anorexia, irritabilidad, dolor abdominal, acompañado de episodios intermitentes de sangrado tipo "jalea de grosella" que evoluciona a sangrado rectal rutilante. El paciente presenta un diagnóstico concomitante de colitis alérgica, lo cual prolonga el tratamiento quirúrgico efectivo en un centro asistencial externo. En la tomografía abdominal se encuentra el signo de la "escarapela" clásico. En la laparotomía exploratoria se confirma invaginación ileoileal, se encuentra una masa que el laboratorio de histopatología confirma como PH. A nuestro conocimiento, es el primer caso de invaginación por $\mathrm{PH}$ pediátrico reportado en el Perú.
\end{abstract}

Palabras clave: Intususcepción; páncreas; Obstrucción intestinal (fuente: DeCS BIREME).

\section{HETEROTOPIC PANCREAS AS A CAUSE OF INTUSSUSCEPTION: FIRST CASE REPORTED IN PERU}

\begin{abstract}
The heterotopic pancreas (HP) is a rare condition in the pediatric population. HP cases involving an ileal intussusception are rare in children and very rarely reported, usually presenting with symptoms of intestinal obstruction. We report the case of a one year old male patient with a chronic history of anorexia, irritability, abdominal pain, accompanied by intermittent episodes of "currant jelly" stools that evolved to rectal bleeding. The patient presented a concomitant diagnosis of allergic colitis, which prolonged the effective surgical treatment at an external health center. In the abdominal CT scan, the classic "target" sign was found. In the exploratory laparotomy an ileoileal intussusception was confirmed, a mass was found that the histopathology laboratory confirmed as HP. To our knowledge, it is the first case of pediatric intussusception by HP reported in Peru.
\end{abstract}

Key words: Intussusception; pancreas; Intestinal obstruction (source: MeSH NLM).

\section{INTRODUCCIÓN}

La invaginación intestinal (II) se define como la introducción de una porción del intestino proximal en el segmento distal contiguo. La II es la causa más común de obstrucción intestinal $(\mathrm{OI})$ en niños entre 3 meses y 6 años, por lo que es necesario que esta enfermedad sea conocida por todo el personal de salud en contacto con pacientes pediátricos ${ }^{(1)}$. A continuación reportamos un caso con presentación atípica de II, causado por una enfermedad muy infrecuente: páncreas heterotópico
$(\mathrm{PH})$ o ectópico. Tras una revisión realizada en los buscadores de Pubmed, SciELO y Lilacs no se encontraron revisiones ni reportes de casos de esta enfermedad en el Perú.

\section{REPORTE DE CASO}

Paciente varón, nacido en Lima, de un año dos meses de edad, sin antecedentes prenatales, médicos o quirúrgicos relevantes. Inicia su enfermedad aparentemente casi

\footnotetext{
Clínica San Felipe. Lima, Perú.

a Cirujano pediatra; ${ }^{\mathrm{b}}$ médico pediatra; ${ }^{\mathrm{c}}$ médico patólogo; ${ }^{\mathrm{d}}$ médico cirujano

Recibido: : 08-04-15 Aprobado: 02-09-15
}

Citar como: Bazán Zender C, Reyes Coloma L, León Cueto JL, Revoredo Palacios G, Arias-Stella Castillo J, Pezo A. Páncreas heterotópico como causa de invaginación intestinal: primer caso reportado en el Perú. Rev Peru Med Exp Salud Publica. 2015;32(3):598-602. 
dos meses antes de su ingreso a nuestra clínica, con anorexia progresiva, irritabilidad, leve distensión abdominal, flatulencia, dolor abdominal moderado y un primer episodio de heces semilíquidas color rojomorado ("jalea de grosella"). Durante una evaluación ambulatoria en otro nosocomio se le encontró Thevenon en heces positivo $(2+)$, leucocitosis $\left(12600 \mathrm{c} / \mathrm{mm}^{3}\right)$ y anemia microcítica e hipocrómica ( $\mathrm{Hb}: 8 ., 2 \mathrm{~g} / \mathrm{dL}$ ). Fue diagnosticado como portador de "alergia alimentaria". Presentó un cuadro respiratorio alto con fiebre, rinorrea y tos, con mejoría de la distensión abdominal, irritabilidad, y ausencia de heces sanguinolentas.

Dieciocho días antes del ingreso presentó un nuevo episodio de heces sanguinolentas con moco, asociados a vómitos alimenticios en tres ocasiones, palidez y persistencia de la anorexia. Se le agregó irritabilidad marcada con deposiciones semilíquidas con moco de color rojo moradas ("jalea de membrillo"), y al presionar el abdomen se evidencio salida de sangre roja rutilante en chorro, por lo que se le hospitalizó en un centro asistencial distinto al nuestro. Durante la hospitalización se realizó una colonoscopía, cuyos hallazgos fueron: "múltiples nodulaciones difusamente distribuidas en todos los segmentos del colon con erosiones y edema moderado". Las biopsias mostraron "mucosa colónica con infiltrado crónico linfocitario y formación de folículos linfoides", lo que llevó al diagnóstico de "colitis nodular alérgica severa" como causa del cuadro. Fue tratado con sintomáticos, hierro y leche sin lactosa y dado de alta con mejoría relativa tras cinco días, los padres refieren pérdida de $1 \mathrm{~kg}$ de peso.

Cinco días antes del ingreso a nuestra clínica presentó un nuevo episodio de deposiciones con moco y rastros sanguinolentos, aumento de distensión abdominal e irritabilidad al ingerir alimentos.

Tras 55 días de enfermedad ingresa a nuestro establecimiento. Al examen, se encuentra un lactante afebril, irritable, en llanto continuo, con abdomen blando, no distendido. A la palpación profunda en el mesogastrio se detecta una masa de aproximadamente $7 \times 6 \mathrm{~cm}$, móvil y dolorosa. Se indican pruebas de laboratorio, con los siguientes resultados relevantes: leucocitos: $16,7 \mathrm{c} / \mathrm{mm}^{3}$; abastonados: 0; Hb: 10,1 g/dL; VSG: 50 mm/h; ferritina: 9,0 ng/mL; anticuerpos gliadina ( $\operatorname{lgA} / \mathrm{lgG})$ : negativos; autoanticuerpos transglutaminasa ( $\lg A)$ : negativo. Los exámenes de PCR, frotis de sangre periférica, VDRL, glucosa, urea, creatinina, transaminasas, GGTP, tiempo de coagulación, tiempo de sangría, y proteinograma sérico estuvieron dentro de límites normales. Una tomografía abdominal contrastada demuestra un asa invaginada coronada por asas libres y una imagen en "escarapela" clásica de II (Figura 1).

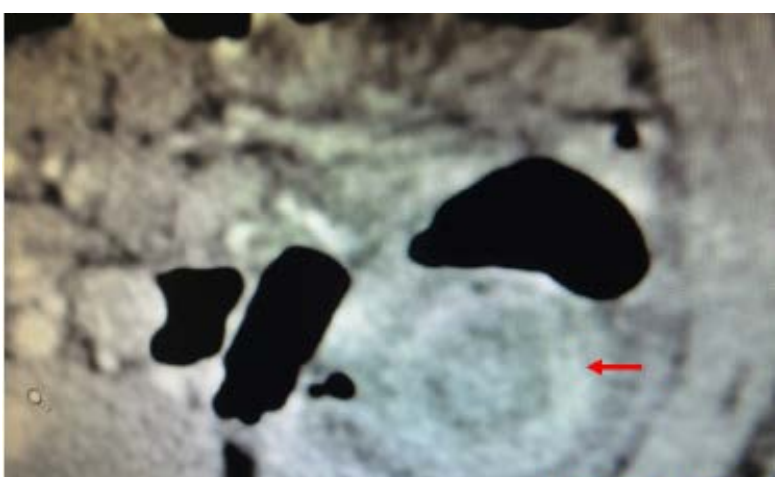

Figura 1. Imagen tomográfica contrastada en "escarapela", también llamada donut o "rosquilla" de la invaginación intestinal del paciente (flecha)

Se realizó laparotomía exploratoria por incisión transversa supraumbilical económica, y se encontró, a $30 \mathrm{~cm}$ de la válvula ileocecal, una doble II íleo-ileal de los segmentos proximal y distal del asa invaginada. Al desinvaginar, se encuentra una pequeña masa dura como eje de la invaginación (Figura 2).

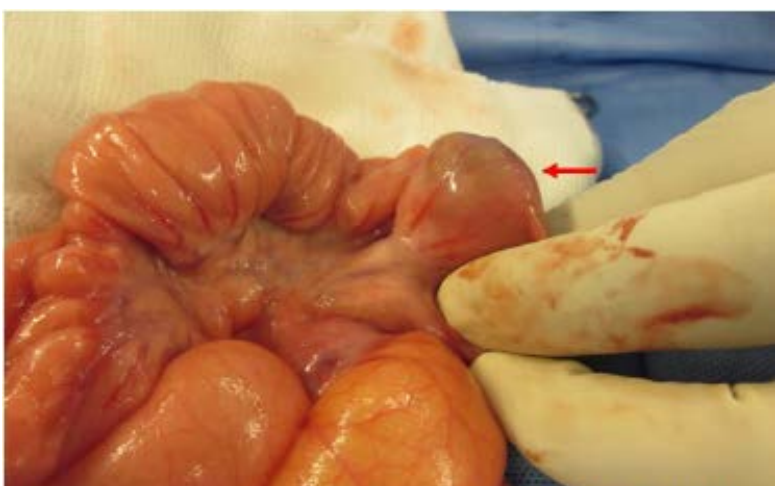

Figura 2. Se aprecia una pequeña masa tumoral de consistencia dura (flecha)

Se realiza la resección de la zona afectada con anastomosis término-terminal. Al invertir la pieza operatoria se encuentra una masa de aspecto polipoide sésil, de $5 \times 2 \mathrm{~cm}$, dura, sólida, con el vértice ulcerado y sangrante (Figura 3).

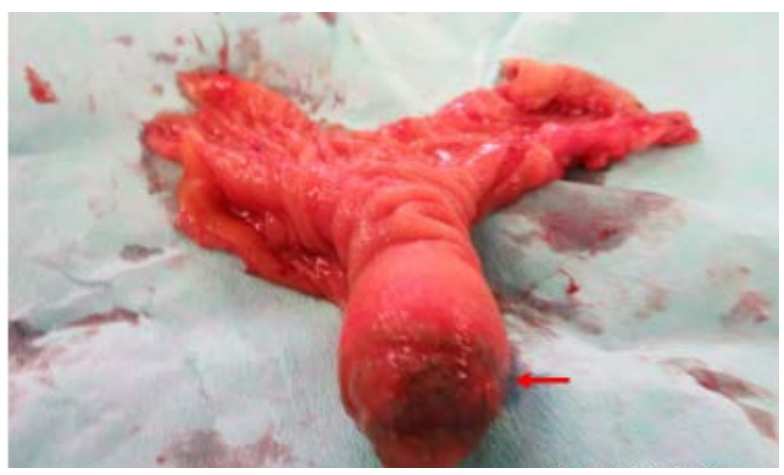

Figura 3. Pieza operatoria evertida mostrando el $\mathrm{PH}$ con el vértice ulcerado y sangrante (flecha) 
El Laboratorio de Histopatología confirmó hallazgos compatibles con un $\mathrm{PH}$, encontraron, en el menor aumento, mucosa ileal normal y mucosa ulcerada vecina al $\mathrm{PH}$, asimismo, presencia de tejido pancreático con desarrollo de ductus pancreáticos distendidos rodeados de ácinos. No se encontraron islotes de Langerhans (Figuras 4 y 5 ).

En el posoperatorio (PO) no se presentaron eventos adversos. Se indicó "nada por vía oral" (NPO) por tres días, seroterapia, antálgicos, ceftriaxona $(75 \mathrm{mg} / \mathrm{kg} /$ día) y clindamicina (10 mg/kg/día). Al PO 2 el dolor abdominal cedió, un hemograma control estuvo dentro de los límites normales con una hemoglobina de $12,5 \mathrm{~g} /$ dL. El dosaje de amilasa y lipasa sérica fue de $69 \mathrm{U} / \mathrm{L}$ y $11 \mathrm{U} / \mathrm{L}$ respectivamente. En el PO 3 se realimentó con líquidos claros, pasando a dieta completa desde el $\mathrm{PO}$ 4 por su buena tolerancia. Fue dado de alta en buenas condiciones en el PO 5. Un control a los cinco meses de la intervención lo encontró asintomático.

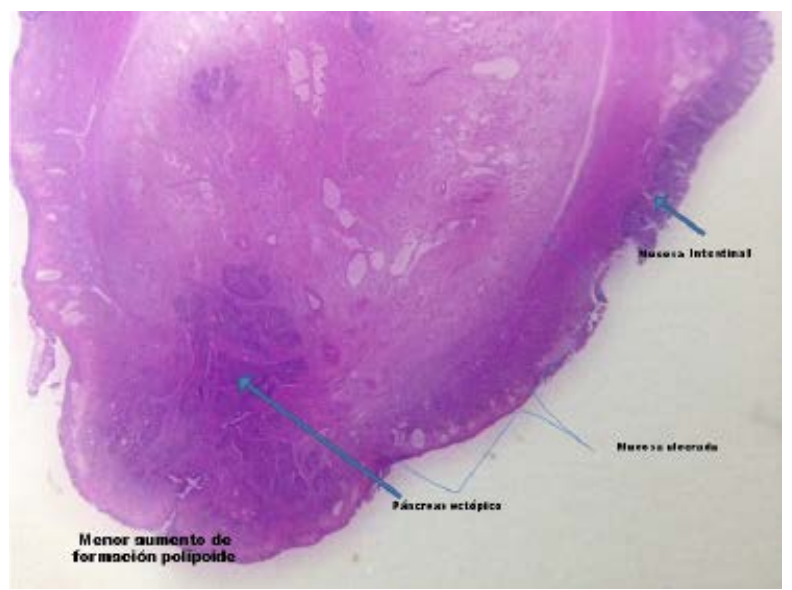

Figura 4. En el menor aumento de la formación polipoide se encuentra mucosa ileal normal y mucosa ulcerada vecina al páncreas heterotópico. Aumento: 5X. Coloración: hematoxilina-eosina

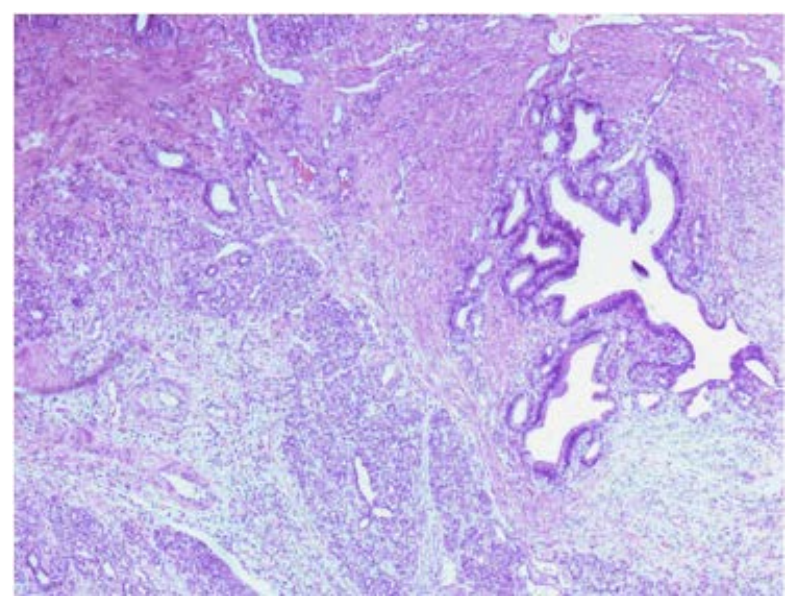

Figura 5. Presencia de conductos y ácinos pancreáticos. Aumento: 10X. Coloración: hematoxilina-eosina

\section{DISCUSIÓN}

La invaginación en la II incluye al mesenterio, cuya compresión provoca un bloqueo del drenaje venoso, edema de la pared e isquemia, produciendo un cuadro de OI. De no tratarse rápidamente puede producir obstrucción arterial y gangrena del intestino proximal ${ }^{(1,2,3)}$. Se manifiesta predominantemente por OI, vómitos, dolor abdominal, sangrado rectal, distensión abdominal y masa palpable ${ }^{(4)}$. Bromberg et al. ${ }^{(5)}$ coincide con Huppertz et al. ${ }^{(6)}$ al encontrar que el sangrado es más común en niños menores de un año, mientras que el dolor abdominal intermitente lo es en niños mayores. Habitualmente, se presenta en niños eutróficos, de forma aguda y grave, siendo menos frecuentes las invaginaciones subagudas y crónicas propias del adulto $y$ de niños mayores ${ }^{(1,4,5-7)}$. Se estima entre 1,5 a 4 casos por 1000 nacidos vivos con una razón hombre:mujer de $3: 2^{(1,2,6,7)}$. La Ol es más frecuente entre los 3 meses a 6 años, con un pico de máxima incidencia entre los 5 y 10 meses ${ }^{(1,3,6)}$. Su localización habitual es alrededor de la válvula ileocecal en el $95 \%$ de los pacientes y es de tipo ileocólica en más del $80 \%$ de los casos. Las restantes pueden ser yeyunoyeyunales, ileoileales o colocolónicas ${ }^{(6,7)}$.

En el Perú, Rosado reporta una serie de 64 niños con II, del Instituto Nacional del Niño (INSN) entre 1973 y 1983, en el cual no se encontraron casos de $\mathrm{PH}^{(7)}$. Jaramillo et al., entre 1997 y 2002, encontraron 34 pacientes con II y solo cuatro invaginaciones ileoileales, ninguna debido a $\mathrm{PH}^{(1)}$. En la mayoría de casos de II no se encuentra causa aparente, catalogándose como idiopática. Las causas conocidas más frecuentes son el divertículo de Meckel (DM), las adenitis mesentéricas, la gastroenterocolitis por rotavirus o adenovirus, la púrpura de Schönlein Henoch y el síndrome de Peutz-Jeghers (SPJ), entre otras ${ }^{(1,3,6,7)}$. Ante la sospecha de II, la ultrasonografía es el método de ayuda diagnóstica de elección, donde el hallazgo es el signo de "la escarapela", también conocido como "rosquilla" o "donut", consistente en la presencia de dos anillos de baja ecogenicidad separados por un anillo hiperecóico ${ }^{(8)}$ patognomónico para realizar el diagnóstico. Se utiliza de manera creciente la tomografía espiral multicorte (TEM) por su gran certeza diagnóstica ${ }^{(9,11)}$. Algunos autores recomiendan la reducción hidrostática o neumática conservadora ${ }^{(8,9)}$ que nosotros no practicamos ya que en nuestro medio se ha reportado recidivas del orden del $10 \%{ }^{(1)}$. Una vez diagnosticado el paciente debe ser intervenido de urgencia por laparotomía abierta o laparoscopía, por el peligro de gangrena y perforación ${ }^{(10)}$.

El PH o páncreas ectópico se define como la presencia de tejido pancreático fuera de su localización anatómica normal, con ausencia de la continuidad vascular y anatómica del páncreas principal $(4,5,11)$. Se origina 
durante la rotación del intestino medio embrionario y la fusión de las partes dorsales y ventrales del páncreas. Debido al íntimo contacto con la parte distal del estómago y el duodeno embrionarios, pequeños islotes de páncreas pueden desprenderse a una localización aberrante, usualmente en el interior de la pared del tracto gastrointestinal ${ }^{(5,11)}$. Ganapathi et al. presentaron un caso de asociación inusual de $\mathrm{PH}$, II ileoileal y ruptura de una banda mesentérica, abriendo la posibilidad de una anomalía en el sistema vascular vitelino como causa ${ }^{(12)}$. Es fundamental para el diagnóstico definitivo encontrar la presencia de tejido pancreático en otro órgano anatómicamente diferente ${ }^{(2,5,11,12)}$.

La histología del $\mathrm{PH}$ está formada por lobulillos de páncreas exocrino, que es infrecuentemente, islotes de Langerhans, que conforman estructuras pancreáticas ductales y acinares, usualmente sin presencia de páncreas endocrino (2,4,5,12). Von Heinrich clasificó histológicamente al $\mathrm{PH}$ en tres tipos: Tipo I en el que se identifican todos los elementos del tejido pancreático; Tipo II en el que se encuentra tejido pancreático con presencia de conductos y ácinos, pero sin islotes; y el Tipo III, caracterizado por ausencia de islotes, muy rara presencia de ácinos y numerosos conductos, algunos de ellos con dilataciones císticas $(13,14)$. El caso que presentamos corresponde al Tipo II ya que concentra ácinos y conductos, pero sin islotes.

Frecuentemente el $\mathrm{PH}$ no causa sintomatología, pero todas las enfermedades de la glándula pueden aquejarlo, incluyendo la pancreatitis crónica, la distrofia quística, y la formación de abscesos y neoplasias ${ }^{(6,12,15,16)}$. El paciente no presentó pancreatitis, evidenciado por los valores normales de enzimas pancreáticas en el PO 2. La presencia de $\mathrm{PH}$ en la II es muy rara considerándose su incidencia entre 1 y $2 \%$ de todos los casos ${ }^{(2,4,5,11)}$. Su localización más frecuente es el tracto gastrointestinal (TGI) proximal, habiéndose descrito casos en la vesícula y vías biliares, bazo, epiplón, mesenterio e incluso mediastino ${ }^{(11,12)}$. Bromberg et al., en una revisión de 18 casos de $\mathrm{PH}$, encontraron siete localizados en el estómago, seis en el duodeno, tres en el yeyuno, uno en la vesícula biliar y uno en un DM, no reportando hallazgos en el íleon ${ }^{(5)}$. Por el contrario, Hamada et al. publicaron tres casos de $\mathrm{PH}$ ileal en II (11). El PH es una entidad totalmente distinta al DM con incorporación de mucosa gástrica y/o pancreática heterotópica, también causante de hematoquezia y presente en el 25 al 56\% de portadores de DM ${ }^{(17)}$.

La II es una enfermedad importante en la población pediátrica, cuya presentación debe ser conocida por el personal de salud para su identificación temprana y tratamiento oportuno. El $\mathrm{PH}$, pese a su extremada rareza, debe estar considerado en el diagnóstico diferencial de lesiones sangrantes del TGI, así como en la presencia de una masa abdominal en el niño. Presentamos el primer reporte de caso de $\mathrm{PH}$ como causa de II en el Perú.

Contribuciones de autoría: CBZ participó en la concepción del artículo, la recolección de datos, su redacción, su revisión crítica y aprobación de la versión final. LRC, JLLC y GR participaron en la concepción del artículo, la recolección de datos, su redacción y aprobación de la versión final. JASC y AP participaron en la recolección de datos, su redacción, revisión crítica del artículo y aprobación de la versión final.

Fuentes de financiamiento: este reporte de caso fue autofinanciado por los autores.

Conflicots de interés: los autores declaran no tener conflictos de interés económico o institucional para la publicación de este reporte de caso.

\section{REFERENCIAS BIBLIOGRÁFICAS}

1. Jaramillo-Sánchez A, JaramilloSamaniego JG. Invaginación Intestinal. En: Jaramillo-Sánchez A, Kuon R, Jaramillo-Samaniego JG, ed. Tratado de cirugía de niños 2014. Lima: Fondo Editorial del Instituto Nacional de Salud del Niño; 2014. p. 359-66.

2. Galván-Montaño A, Suárez-Roa M de L, Estrada-Hernández M del R. [Jejunal intussusceptions as a lead point ectopic pancreatic tissue in a 1-year-old male. Case report]. Cir Cir. 2012;80(6):5469. [Artículo en español]

3. Waseem M, Rosenberg HK. Intussusception. Pediatr Emerg Care.
2008;24(11):793-800. doi: 10.1097/ PEC.0b013e31818c2a3e.

4. Singh S, Batra A, Sangwaiya A, Marwah N, Rattan K, Sen R. Heterotopic Pancreas presenting as ileoileal intussusception. J Surg Case Rep. 2012;2012(9):13. doi: 10.1093/jscr/2012.9.13.

5. Bromberg SH, Camilo Neto C, Borges AF, Franco MI, França LC, Yamaguchi N. Pancreatic heterotopias: clinicopathological analysis of 18 patients. Rev Col Bras Cir. 2010;37(6):413-9.

6. Huppertz HI, Soriano-Gabarró M, Grimpel E, Franco E, Mezner Z, Desselberger $\mathrm{U}$, et al. Intussusception among young children in Europe. Pediatr Infect Dis J. 2006;25(1 Suppl):S22-9.

7. Rosado-Bejarano F. Invaginación intestinal. En: Rivas C. Abdomen agudo. 1ra ed. Lima: Integral; 1990. p. 39-45.

8. Yip WC, Ho TF, Yip YY, Chan KY. Value of abdominal sonography in the assessment of children with abdominal pain. J Clin Ultrasound.1998;26(8):397-400.

9. Ko HS, Schenk JP, Tröger J, Rohrschneider WK. Current radiological management of intussusception in children. Eur Radiol. 2007;17(9):2411-21. 
10. Apelt N, Featherstone N, Giuliani S. Laparoscopic treatment of intussusception in children: a systematic review. J Pediat Surg. 2013;48(8):1789-93. doi: 10.1016/j. jpedsurg.2013.05.024.

11. Hamada Y, Yonekura Y, Tanano A, Takada K, Kato Y, Sato M, et al. Isolated heterotopic pancreas causing intussusception. Eur J Pediatr Surg. 2000;10(3):197-200.

12. Ganapathi S, Villa F, Perera R, Wan A. Ectopic pancreas, intussusception, and a ruptured mesenteric band: an unusual association. Clin Anat. 2011;24(1):128-32. doi: 10.1002/ ca.21052..

13. VermaS, Bansal P, Manchanda V, Gupta $\mathrm{R}$. Isolated ileal heterotopic pancreas in a child: A clinically undetected cause of ileoileal intussusception. International Journal of Case Reports and Images. 2014;5(2):122-5.

14. Inoue $\mathrm{Y}$, Hayashi M, Arisaka Y, Higuchi K, Egashira Y, Tanigawa $\mathrm{N}$. Adenocarcinoma arising in a heterotopic pancreas (Heinrich type III): a case report. J Med Case Rep. 2010;4:39.

15. Zhang L, Sanderson SO, Lloyd RV, Smyrk TC. Pancreatic intraepithelial neoplasia in heterotopic pancreas evidence for the progression model of pancreatic ductal adenocarcinoma. Am J Surg Pathol. 2007;31(8):1191-5.

16. Esfahani H, Olad E, Dehghan A, Bazmamoun $\mathrm{H}, \quad$ Ghorbanpoor M. Infantile extrapancreatic pancreatoblastoma: a report on a rare infantile abdominal mass. J Pediatr Hematol Oncol. 2014;36(3):241-5. doi: 10.1097/ MPH.0b013e3182a0627f.

17. Llanos-Rodríguez R. Divertículo de Meckel. En: Jaramillo-Sánchez A, Kuon R. Tratado de cirugía pediátrica. 1ra ed. Lima: CONCYTEC; 2006. p. $352-5$.

Correspondencia: Carlos Bazán Zender Dirección: Av. Gregorio Escobedo 650, Jesus María. Lima, Perú

Teléfono: (+511) 991321000

Correoelectrónico:mariabeltranfa@gmail.com

\section{http://www.ins.gob.pe/rpmesp}

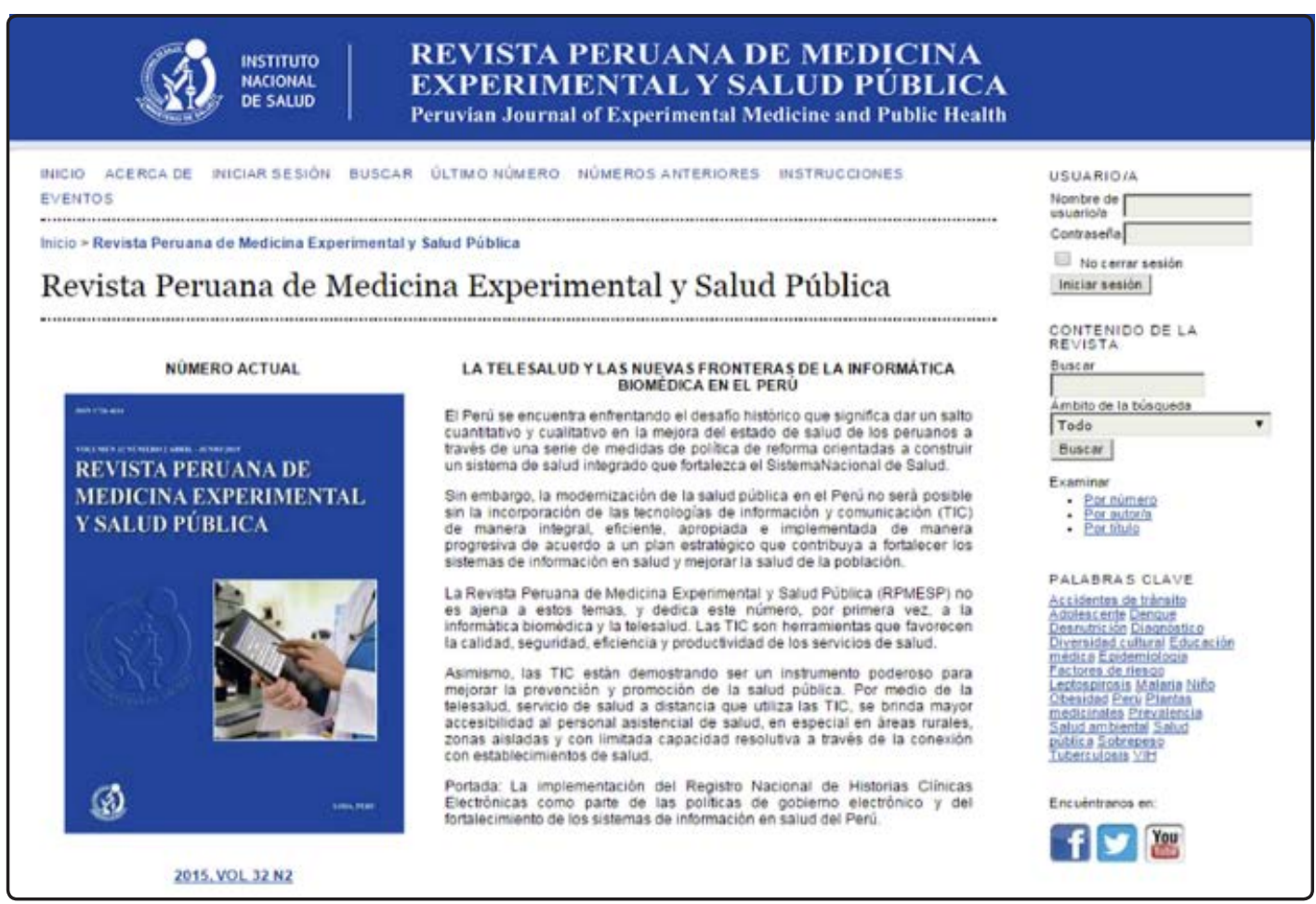

